\title{
Atrial low voltage areas: A comparison between atrial fibrillation and sinus rhythm
}

\author{
Ana Andrés Lahuerta ${ }^{1,2}$, Carlos Roberto ${ }^{3}$, Francisco Javier Saiz ${ }^{3}$, \\ Óscar Cano ${ }^{2}$, Laura Martínez-Mateu³, Pau Alonso², \\ Assumpció Saurí ${ }^{2}$, Aurelio Quesada ${ }^{1}$, Joaquín Osca ${ }^{2}$ \\ ${ }^{1}$ Escuela de Doctorado, Universidad Católica de Valencia San Vicente Mártir, Spain \\ ${ }^{2}$ Unidad de Arritmias, Hospital Universitario y Politécnico La Fe, Valencia, Spain \\ ${ }^{3}$ Universitat Politècnica de València, Spain
}

\begin{abstract}
Background: Atrial fibrosis can promote atrial fibrillation (AF). Electroanatomic mapping (EAM) can provide information regarding local voltage abnormalities that may be used as a surrogate marker for fibrosis. Specific voltage cut-off values have been reproduced accurately to identify fibrosis in the ventricles, but these values are not well defined in atrial tissue.

Methods: This study is a prospective single-center study. Patients with persistent AF referred for ablation were included. EAM was performed before ablation. We recorded bipolar signals, first in AF and later in sinus rhythm (SR). Two thresholds delimited low-voltage areas (LVA), 0.5 and $0.3 \mathrm{mV}$. We compared LVA extension between maps in SR and AF in each patient.

Results: A total of 23 patients were included in the study. The percentage of points with voltage lower than $0.5 \mathrm{mV}$ and $0.3 \mathrm{mV}$ was significantly higher in maps in AF compared with maps in SR: $38.2 \%$ of points $<0.5 \mathrm{mV}$ in $A F$ vs. $22.9 \%$ in $S R(p<0.001) ; 22.3 \%$ of points $<0.3 \mathrm{mV}$ in $A F$ vs. $14 \%$ in $S R(p<0.001)$. Areas with reduced voltage were significantly larger in maps in $A F(0.5 \mathrm{mV}$ threshold, mean area in $A F 41.3 \pm 42.5 \mathrm{~cm}^{2}$ vs. $11.7 \pm 17.9 \mathrm{~cm}^{2}$ in $S R, p<0.001 ; 0.3 \mathrm{mV}$ threshold, mean area in $A F 15.6 \pm 22.1 \mathrm{~cm}^{2}$ vs. $6.2 \pm 11.5 \mathrm{~cm}^{2}$ in $\left.S R, p<0.001\right)$.

Conclusions: Using the same voltage thresholds, LVA extension in AF is greater than in SR in patients with persistent $A F$. These findings provide arguments for defining a different atrial fibrosis threshold based on EAM rhythm. (Cardiol J 2022; 29, 2: 252-262)
\end{abstract}

Key words: atrial fibrillation, electroanatomic mapping, low-voltage areas, atrial fibrosis threshold, pulmonary vein isolation

\section{Introduction}

Atrial fibrillation (AF) is the most common cardiac tachyarrhythmia. The pathophysiology of AF is complex [1] and can be described as follows [2]: 1) triggers for the initiation of arrhythmia (pulmonary and nonpulmonary foci); 2) a fibrotic substrate for the maintenance of AF; and 3) various modulators acting by multiple potential mechanisms (such as hypertension, obesity, obstructive sleep apnea, inflammation, and endurance sports).

Atrial remodeling and fibrosis development are associated with a variety of electric disturbances, such as heterogeneities in atrial action potential

Address for correspondence: Ana Andrés Lahuerta, C/Maestro Palau 12, 7-14. CP 46008, Valencia, Spain, tel: +34690100553, e-mail: anaandreslahuerta@gmail.com

Received: 9.05.2021 Accepted: 17.08.2021 Early publication date: 18.10.2021

This article is available in open access under Creative Common Attribution-Non-Commercial-No Derivatives 4.0 International (CC BY-NC-ND 4.0) license, allowing to download articles and share them with others as long as they credit the authors and the publisher, but without permission to change them in any way or use them commercially. 
duration, effective refractory period, and conduction velocity [3]. These phenomena can promote and sustain $\mathrm{AF}$.

Left atrial (LA) scarring can be detected by late enhancement magnetic resonance imaging (MRI-DE) and can be correlated well with reduced electrogram amplitudes as recorded by endocardial voltage maps [4]. However, MRI to assess atrial fibrosis is not available in all centers because of its complex evaluation. Electroanatomic mapping (EAM) can provide information regarding local voltage abnormalities that may be used as a surrogate marker for fibrosis. Specific voltage cut-off values have been reproducibly shown accurately to identify scars and/or fibrosis in the ventricles $[5,6]$. EAM voltage cut-off values to identify myocardial scars in the atrial tissue are not as well defined as in the ventricle. Voltage-guided AF substrate modification targeting low voltage areas (LVAs) has been carried out in some studies to improve long-term $\mathrm{AF}$ ablation efficacy [7-12]. In most of them, mapping was performed using voltage cut-offs during sinus rhythm (SR), and in one study, mapping was performed during AF [12]. However, a recent study documented that the correlation between lowvoltage and posterior LA MRI-DE is significantly improved when acquired during AF vs. SR [13]. All of these studies used a cut-off point of $0.5 \mathrm{mV}$ to define low voltage, although some of them also used other cut-off points such as 0.1 or $0.2 \mathrm{mV}$ to define the scar area. Some of them used a $3.5 \mathrm{~mm}$ saline-irrigated catheter for mapping [8, 9], Jadidi et al. [10] used a 20-pole mapping catheter whereas others studies combined two types of catheters, a decapolar or a 20-pole mapping catheter and a $3.5 \mathrm{~mm}$ saline-irrigated catheter $[7,11]$.

The aim of the present study was to evaluate the LVA extent and location in patients with persistent $\mathrm{AF}$ undergoing pulmonary vein isolation (PVI) and to compare the findings on maps obtained in $\mathrm{SR}$ and $\mathrm{AF}$ in each patient.

\section{Methods}

This study was a prospective single-center study. Patient demographics, clinical characteristics, and medications were exported from patient records. All participants provided written informed consent for the ablation procedure and for inclusion in medical research at the time of the procedure.

\section{Study population}

All consecutive patients with persistent AF referred to the documented center for PVI were included (between September 2016 and June 2019). Exclusion criteria were redo ablations (to avoid bias owing to prior ablations, which could affect $\mathrm{AF}$ substrate) and patients who arrived in SR on the procedure day.

\section{Electroanatomic mapping and ablation}

All procedures were performed under general anesthesia. Antiarrhythmic agents, if present, were not discontinued. Patients maintained anticoagulant treatment (acenocumarol with an international normalized ratio from 2 to 3.5, or direct oral anticoagulants, with the last dose the night before the procedure).

After vascular access was obtained, a double transseptal puncture was performed, and intravenous heparin was administered to maintain an activated clotting time of more than $300 \mathrm{~s}$. Thereafter, two long sheaths (1 SL0 sheath and 1 Agilis sheath; St. Jude Medical, Inc., St. Paul, MN) were inserted into the LA. The following catheters were used: a decapolar catheter WEBSTER ${ }^{\circledR}$ CS Catheter (Biosense Webster) was placed in the coronary sinus as a reference (6 Fr), a Pentaray ${ }^{\circledR}$ catheter (Biosense Webster) with F curve 2-6-2 mm spacing between electrodes ( $7 \mathrm{Fr}$ ) as a high-density mapping catheter (10 pairs of electrodes) and a SmartTouch SF with an F curve as an irrigated-tip contact force ablation catheter (7.5 Fr).

The three-dimensional geometry of the LA and 4 pulmonary veins (PVs) was reconstructed with the use of the Carto3 mapping system version 6 (Biosense Webster, Inc.). To ensure that the mapping catheter was in contact with the tissue, the CARTO system features the TPI or tissue proximity indicator, which performs an impedance matrix. When it contacts the cardiac wall, the catheter has less ion-charged blood, so impedance rises. To carry out the automatic acquisition of points, a series of filters were included. Multiple bipolar signals (filter setting: $30-300 \mathrm{~Hz}$ ) were recorded from the Pentaray catheter, first in AF and later in SR after electrical cardioversion. Operators mapped carefully to ensure that the entire LA anatomy was represented in both the SR and AF maps. All points within the PVs and LA appendage were excluded. After both electroanatomical maps were completed, ablation was performed as usual in our center: ipsilateral PVI in pairs, with entrance and exit block as the electrophysiological endpoint. In some cases, ablation could also include lines of ablation at the operator's discretion.

All procedures were performed by two expert operators. 


\section{Fibrosis area measurement}

Two thresholds, selected according to the literature, were established to delimit LVA: $0.5 \mathrm{mV}$ and $0.3 \mathrm{mV}$. The condition of presenting voltages higher than or equal to $0.1 \mathrm{mV}$ was added to avoid areas of dense fibrosis and possible false voltages, which might be due to contact problems or which may represent electrical noise.

To delimitate LVA extension, a tool present in CARTO called "area measurement" was used. With this tool, low voltage extension is manually drawn according to the color code given by the chosen thresholds. Since this measurement process is performed manually, there may be discrepancies between one measurement and another. To eliminate variability, two observers made two measurements of each patient.

\section{Qualitative score of fibrosis distribution in $\mathrm{AF}$ and $\mathrm{SR}$}

To correlate the location of the LVA between the AF and SR maps, a qualitative score was defined based on 4 projections (antero-posterior [AP], postero-anterior [PA], superior [SUP] and inferior [INF]). In every view, LVA in SR and AF were compared, assigning different values according to the degree of similarity. In this way, a value of 0 would correspond to discrepancies in the location of LVA between both maps; if LVA were in the same zones, the assigned value would be 2 . For cases where there was no LVA in SR and therefore the comparison was not possible, the assigned value would be 1 . Finally, values obtained for each projection were added, obtaining a final value. If this value was equal to or greater than 5 , it was defined as correspondence between LVA in both maps. Thus, patients in whom the location of the LVA differs in at least two projections are considered mismatched. This process was performed for both thresholds, 0.5 and $0.3 \mathrm{mV}$.

\section{Follow-up}

Antiarrhythmic medications were continued based on medical criteria. A blanking period of 3 months after the ablation procedure was considered during which arrhythmia recurrence was not judged as ablation failure. All patients underwent ambulatory medical control visits 6 and 12 months after the ablation procedure. At each time point, a 12-lead electrocardiogram (ECG) and 24-hour Holter ECG were recorded. If patients presented symptoms earlier, they underwent additional 12-lead ECG and 24-hour Holter ECG. Arrhythmic recurrence at least one episode of sustained $\mathrm{AF}$
Table 1. Clinical characteristics $(n=23)$.

\begin{tabular}{lc}
\hline Clinical characteristics & \\
Age [years] & $59.2 \pm 7.6$ \\
Sex (male) & $74 \%(n=17)$ \\
AF time of evolution & $4.5 \pm 3.8$ \\
[years] & \\
Hypertension & $52 \%(n=12)$ \\
Diabetes mellitus & $9 \%(n=2)$ \\
Dyslipidemia & $39 \%(n=5)$ \\
Smoking & $39 \%(n=9)$ \\
Sleep apnea syndrome & $13 \%(n=3)$ \\
Left atrium enlargement & $87 \%(n=20)$ \\
Heart disease & $35 \%(n=8)$ \\
Treatment & \\
ACO & $95.7 \%(n=22)$ \\
AA & $69.6 \%(n=16)$ \\
BB & $87 \%(n=20)$ \\
CA & $4.3 \%(n=1)$ \\
\hline
\end{tabular}

Left atrium enlargement includes an anteroposterior diameter greater than $40 \mathrm{~mm}$ or an indexed volume greater than $35 \mathrm{~mL} / \mathrm{m}^{2}$ by echocardiography or an indexed volume greater than $53 \mathrm{~mL} / \mathrm{m}^{2}$ by cardiac magnetic resonance; $\mathrm{AF}$ - atrial fibrillation; $\mathrm{ACO}-$ oral anticoagulant treatment; $A A-$ antiarrhythmic treatment (it includes amiodarone, flecainide, propafenone and dronedarone); $\mathrm{BB}$ - beta-blockers treatment; $\mathrm{CA}$ - non-dihydropyridine calcium channel blockers treatment

or atrial flutter ( $>30 \mathrm{~s})$ was considered and was recorded on a surface 12-lead ECG or Holter ECG.

\section{Statistical analysis}

The IBM SPSS statistics package was used for statistical analysis. Numerical data were tested for normality using the Shapiro-Wilk test. Numerical variables are expressed as the mean \pm standard deviation. Categorical variables are expressed in percentages. Paired and unpaired t tests were used when appropriate. Categorical variables were compared between groups by using the $\chi^{2}$ test. The significance level was defined as $\mathrm{p}<0.05$. To evaluate interobserver concordance, the intraclass correlation coefficient was calculated.

Kaplan-Meier curves were created for the endpoint of arrhythmia-free survival. Arrhythmia-free survival was then compared between the groups using the log-rank test.

\section{Results}

A total of 23 patients were included. Patient characteristics are shown in Table 1 . All the studied variables had a normal distribution by the Shapiro-Wilk test. 


\section{Procedure}

All patients were in AF before the procedure. Acute PVI was achieved in all patients. Two of them also had a history of common isthmus-dependent atrial flutter, so cavotricuspid isthmus ablation was also performed. Ablation lines were performed in 3 patients who presented atypical left atrial flutter during the procedure.

The median procedure time (from femoral venous puncture to removal of all catheters) was $169.3 \pm 29 \mathrm{~min}$, and the median fluoroscopy time was $5.6 \pm 2.5 \mathrm{~min}$.

The only complication derived from the procedure was one case of cardiac tamponade (4.3\%), which was solved by pericardiocentesis.

\section{Electroanatomic mapping and low voltage area study}

Number and percentage of points. The mean number of points for maps in AF was $3428 \pm$ \pm 1159 points, whereas it was $2319 \pm 1143$ points for maps in SR.

Percentage of points with voltage lower than $0.5 \mathrm{mV}$ and with voltage lower than $0.3 \mathrm{mV}$ was significantly higher in maps in $\mathrm{AF}$ compared to maps in SR: $38.2 \%$ of points $<0.5 \mathrm{mV}$ in maps in AF vs. $22.9 \%$ of points $<0.5 \mathrm{mV}$ in maps in SR ( $\mathrm{p}<0.001$ ); $22.3 \%$ of points $<0.3 \mathrm{mV}$ in maps in AF vs. $14 \%$ of points $<0.3 \mathrm{mV}$ in maps in SR $(\mathrm{p}<0.001)$.

Mean voltage. The global mean voltage of maps in AF was significantly lower than the mean voltage of maps in SR: $0.62 \pm 0.27 \mathrm{mV}$ vs. $1.62 \pm$ $\pm 0.7 \mathrm{mV}(\mathrm{p}<0.001)$. When areas with voltages lower than $0.5 \mathrm{mV}$ were selected, significant differences in mean voltage were not observed between the two types of maps $(0.277 \pm 0.02 \mathrm{mV}$ vs. $0.272 \pm$ $\pm 0.01 \mathrm{mV}, \mathrm{p}=0.27)$. No differences were found in the mean voltage in areas with voltages lower than $0.3 \mathrm{mV}(0.192 \pm 0.008 \mathrm{mV}$ in maps in AF vs. $0.92 \pm 0.007 \mathrm{mV}$ in maps in SR, $\mathrm{p}=0.448$ ).

Low voltage area measurement. For the $0.5 \mathrm{mV}$ threshold, all patients had LVA when the maps were performed in AF, whereas only $52.2 \%$ of patients had LVA when the maps were performed in SR. For the $0.3 \mathrm{mV}$ threshold, $78.2 \%$ of patients had LVA in the AF maps, whereas only $43.5 \%$ of patients had LVA in the SR maps.

Although the global area of maps in $\mathrm{AF}$ was smaller than that of maps in SR $\left(267.9 \pm 44.5 \mathrm{~cm}^{2}\right.$ vs. $285.7 \pm 48.2 \mathrm{~cm}^{2}, \mathrm{p}<0.001$ ), areas with reduced voltages were significantly larger in maps in AF. Specifically, for the $0.5 \mathrm{mV}$ threshold, the mean LVA in AF maps was $41.3 \pm 42.5 \mathrm{~cm}^{2}$, whereas in

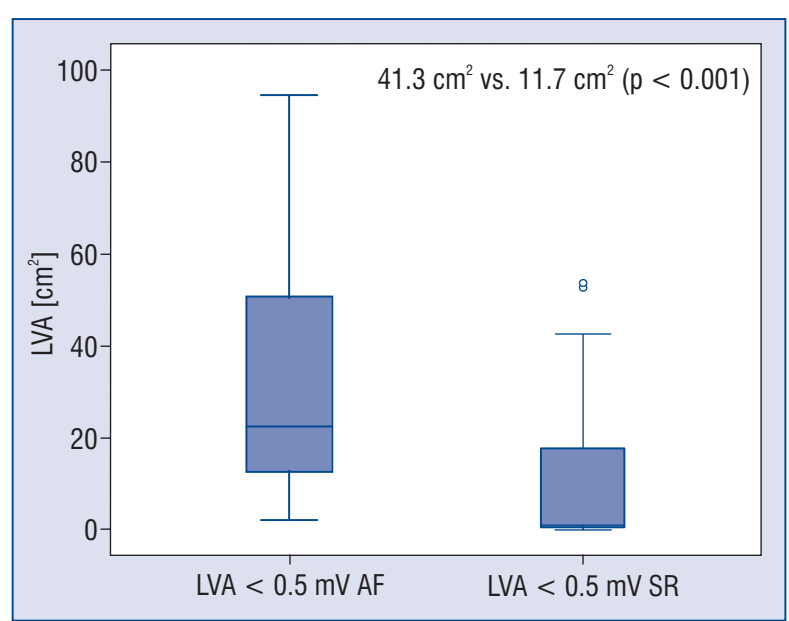

Figure 1. Low voltage area (LVA) extension $<0.5 \mathrm{mV}$ : comparison between maps in atrial fibrillation (AF) and maps in sinus rhythm (SR).

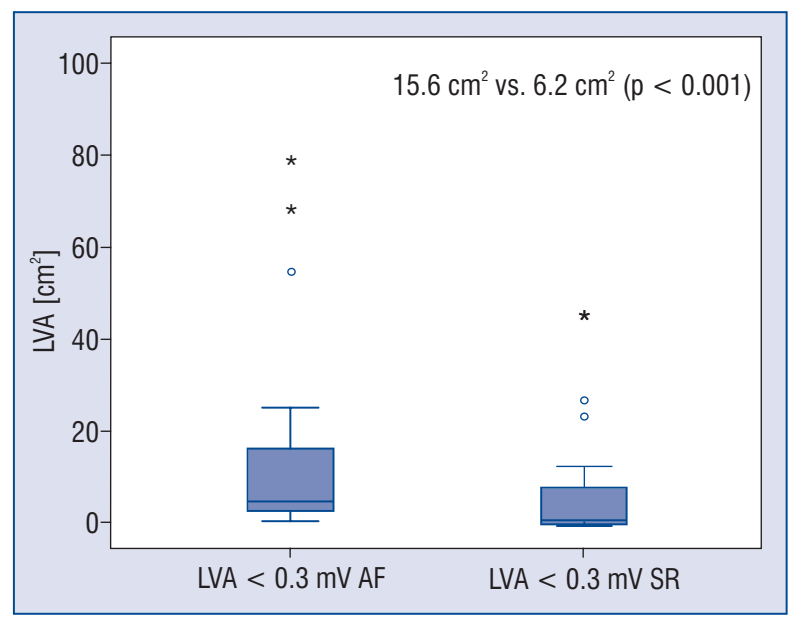

Figure 2. Low voltage area (LVA) extension < $0.3 \mathrm{mV}$ : comparison between maps in atrial fibrillation (AF) and maps in sinus rhythm (SR).

maps in $\mathrm{SR}$, it was $11.7 \pm 17.9 \mathrm{~cm}^{2}, \mathrm{p}<0.001$. For the $0.3 \mathrm{mV}$ threshold, the mean LVA in maps in AF was $15.6 \pm 22.1 \mathrm{~cm}^{2}$, whereas it was $6.2 \pm 11.5$ $\mathrm{cm}^{2}$ in maps in SR, $\mathrm{p}<0.001$ (Figs. 1, 2).

\section{Qualitative score of assessment of fibrosis}

For the $0.5 \mathrm{mV}$ threshold, $91 \%$ of patients obtained a score equal to or greater than 5 points, which, considering the description of our score, means that LVA in SR was present as LVA in maps in AF. The remaining 9\% did not have LVA in SR maps, so location comparison could not be made. For the $0.3 \mathrm{mV}$ threshold, $52 \%$ of patients obtained 


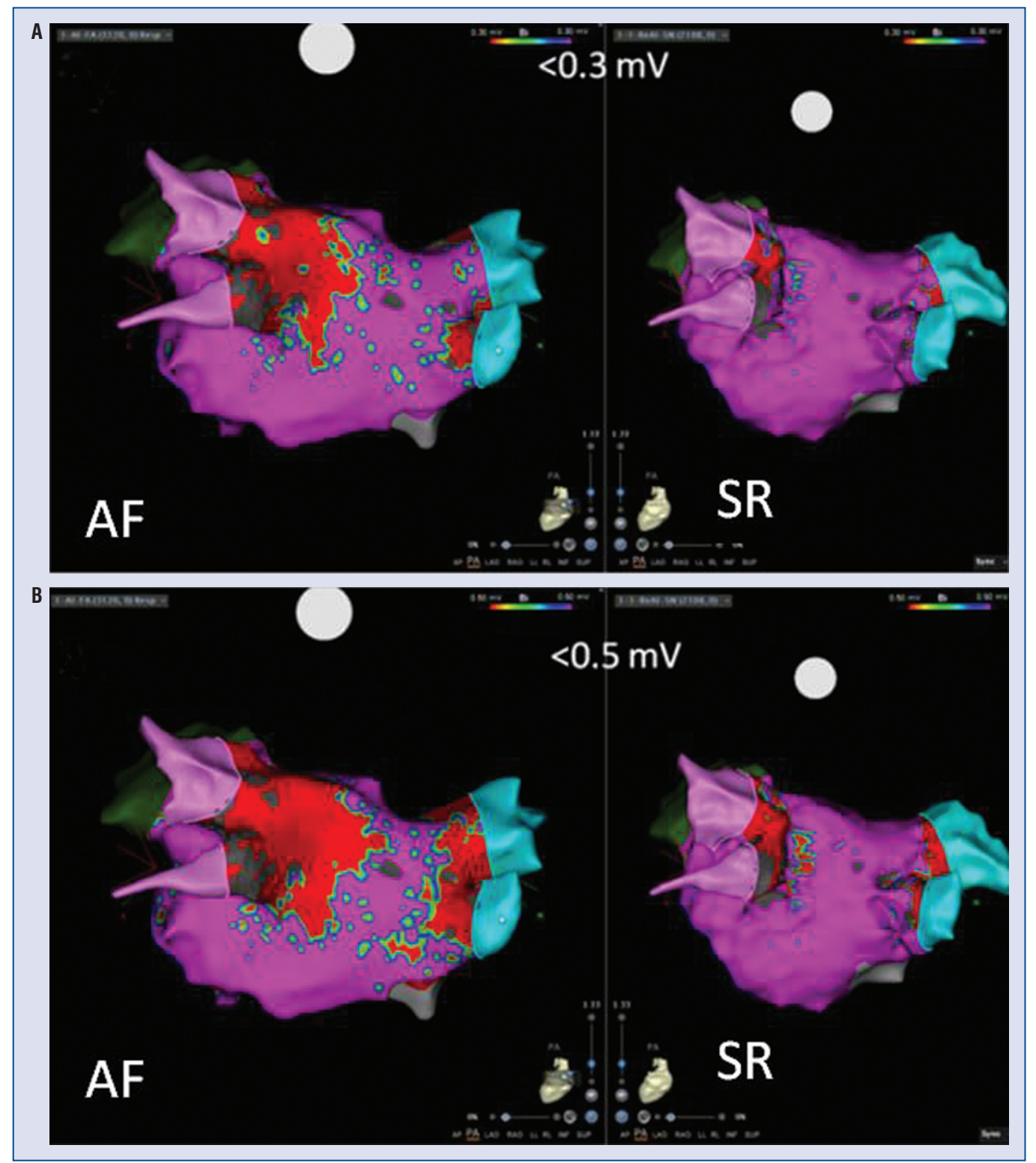

Figure 3. A. Comparison between atrial fibrillation (AF) and sinus rhythm (SR) maps for the $0.3 \mathrm{mV}$ threshold. Postero-anterior projection. Patient 1; B. Comparison between AF and SR maps for the $0.5 \mathrm{mV}$ threshold. Postero-anterior projection. Patient 1. In both images, an appropiate match in low voltage area (LVA) location was observed, although LVA extension was greater in the AF map.

a score equal to or greater than 5 points, which, considering our score, meant that LVA in SR was present in AF. In 39\% of patients, there was no LVA in maps in SR, so location comparison could not be made. Finally, in the remaining $9 \%$ of patients, the score was less than 4 points, so LVA in SR was not represented as LVA in AF (Figs. 3A, B; 4A, B; $5 \mathrm{~A}, \mathrm{~B} ; 6 \mathrm{~A}, \mathrm{~B})$.

Interobserver correlation. To assess interobserver correlation, the intraclass correlation coefficient was calculated based on the measure- ments carried out for each patient and each area by two independent observers. The results showed an adequate correlation with values greater than 0.8 in all cases: (i) Area measurement $\mathrm{AF}<0.5 \mathrm{mV}$ : intraclass correlation coefficient $0.88, \mathrm{p}<0.001$; (ii) Area measurement $\mathrm{AF}<0.3 \mathrm{mV}$ : intraclass correlation coefficient 0.92, $\mathrm{p}<0.001$; (iii) Area measurement $\mathrm{SR}<0.5 \mathrm{mV}$ : intraclass correlation coefficient 0.89, $\mathrm{p}<0.001$; (iv) Area measurement $\mathrm{SR}<0.3 \mathrm{mV}$ : intraclass correlation coefficient $0.86, \mathrm{p}<0.001$. 


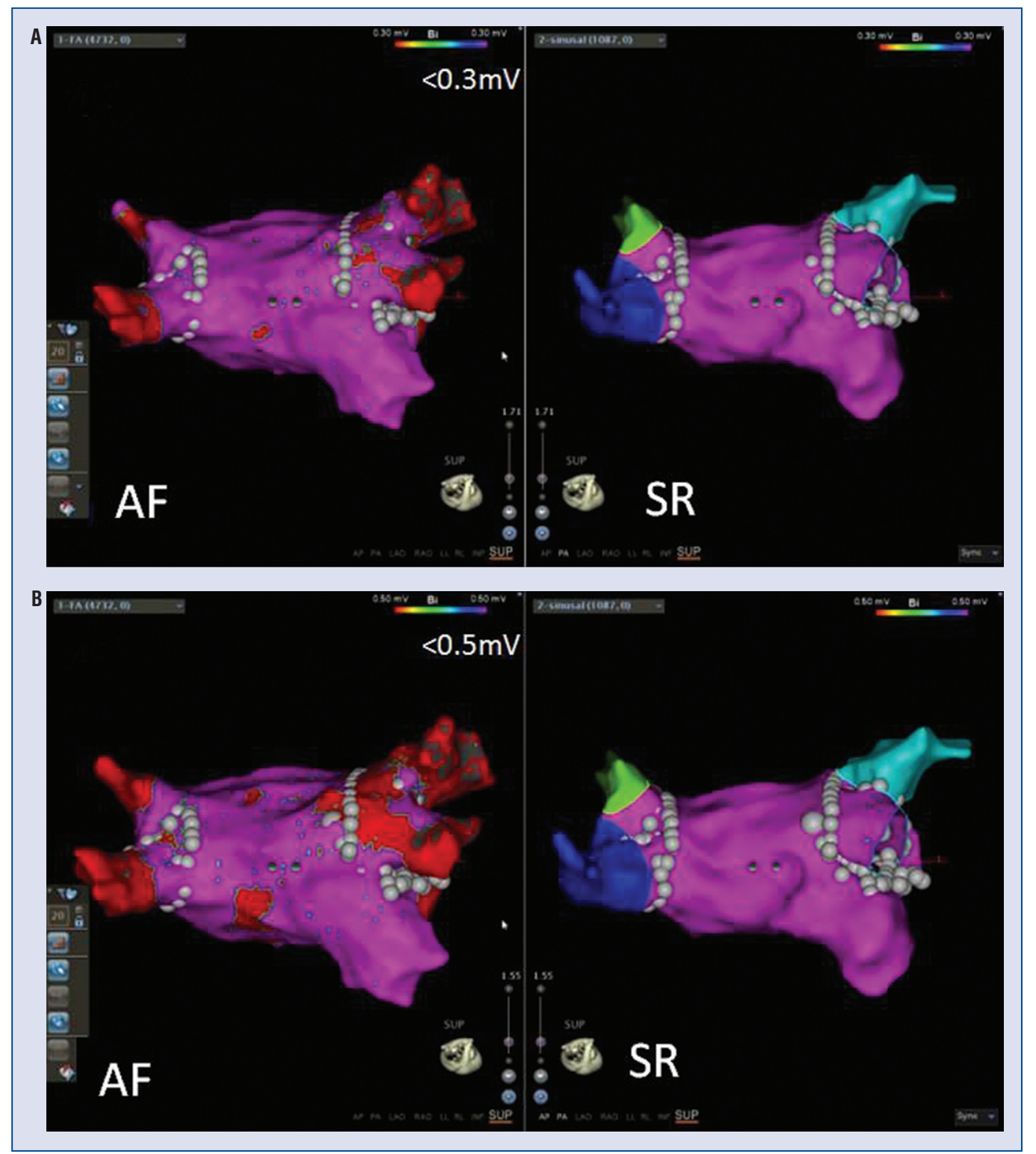

Figure 4. A. Comparison between atrial fibrillation (AF) and sinus rhythm (SR) maps for the $0.3 \mathrm{mV}$ threshold. Superior projection. Patient 2; B. Comparison between AF and SR maps for the $0.5 \mathrm{mV}$ threshold. Superior projection. Patient 2. In both images, lack of low voltage area (LVA) in SR maps is observed, for $0.3 \mathrm{mV}$ threshold as well as for the $0.5 \mathrm{mV}$ threshold. In this case it is not possible to assess if LVA location matches appropriately.

\section{Follow-up}

The mean follow-up time was $25 \pm 12$ months. Excluding the blanking period, 10 patients (43.5\% of all patients) presented arrhythmic recurrence at the postablation follow-up. Among patients who had recurrence, 8 presented $\mathrm{AF}$ ( $80 \%$ of the patients with recurrence), whereas 2 presented atrial flutter (20\% of the patients with recurrence).

The median arrhythmia-free survival at followup was $25.3 \pm 3.7$ months.
In the global sample, for the $0.5 \mathrm{mV}$ threshold, the mean percentage of LVA in maps in SR was $4.2 \%$, whereas it was $15.6 \%$ in maps in $\mathrm{AF}$. For the $0.3 \mathrm{mV}$ threshold, the mean percentage of LVA in maps in SR was $2.2 \%$, whereas it was $5.8 \%$ in maps in AF. The relationship between the percentage of LVA and arrhythmic recurrences was studied. The statistical power of the study was too low to detect significant differences in LVA according to the presence of recurrences, 


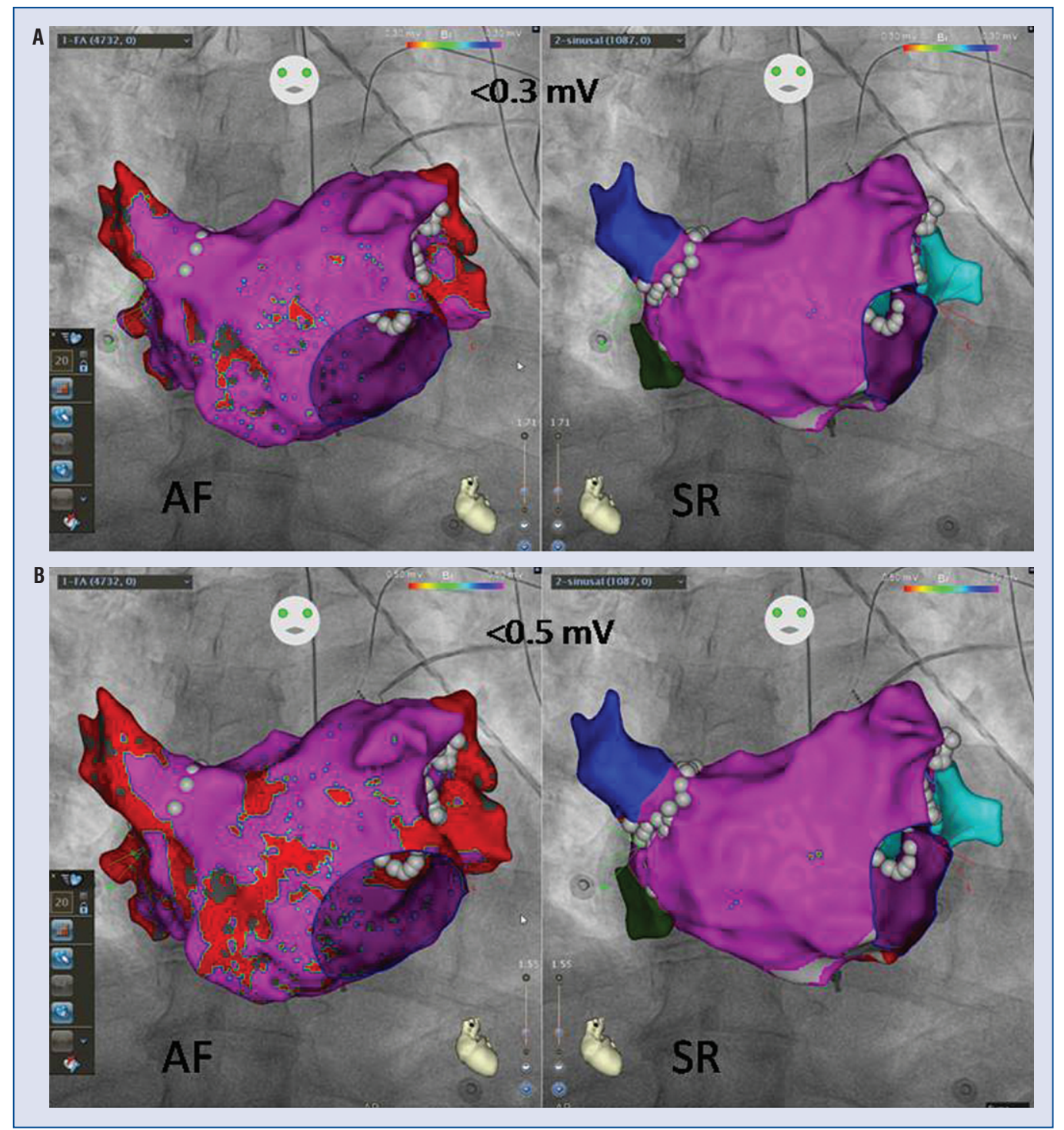

Figure 5. A. Comparison between atrial fibrillation (AF) and sinus rhythm (SR) maps for the $0.3 \mathrm{mV}$ threshold. Anteroposterior projection. Patient 5; B. Comparison between atrial fibrillation (AF) and sinus rhythm (SR) maps for the $0.5 \mathrm{mV}$ threshold. Anteroposterior projection. Patient 5. In both images, low voltage area (LVA) extension in AF is much greater than in SR.

so the present results must be considered exploratory. For the $0.5 \mathrm{mV}$ AF, patients with recurrences presented a greater percentage of LVA than patients without recurrences $(19.1 \%$ vs. $9.8 \%$, $\mathrm{p}=0.019)$. For the same threshold, the SR result was not significant $(5.5 \%$ vs. $3.1 \%, \mathrm{p}=0.381)$. For the $0.3 \mathrm{mV}$ group, the percentage of fibrosis did not show a significant relationship with arrhythmic recurrences in either AF or SR $(6.75 \%$ vs. $3.1 \%$, $\mathrm{p}=0.738,2.6 \%$ vs. $1.9 \%, \mathrm{p}=0.952$ ).

According to receiver operating characteristic curves (Fig. 7A) and to fibrosis stage I definition in previous articles [14], we studied the relationship of the percentage of LVA $<0.5 \mathrm{mV}$ greater than $5 \%$ with arrhythmic recurrences. In AF, both variables showed an almost significant association $(\mathrm{p}=0.06)$ : $56.3 \%$ of patients that presented a percentage of LVA greater than 5\% had arrhythmic recurrences whereas only $14.3 \%$ of patients with a percentage of LVA less than 5\% had a recurrence (Fig. 7B). In SR, the result was not significant $(\mathrm{p}=0.382)$.

\section{Discussion}

Low voltage area studied by EAM of atrial bipolar endocardial signals has been recently estab- 


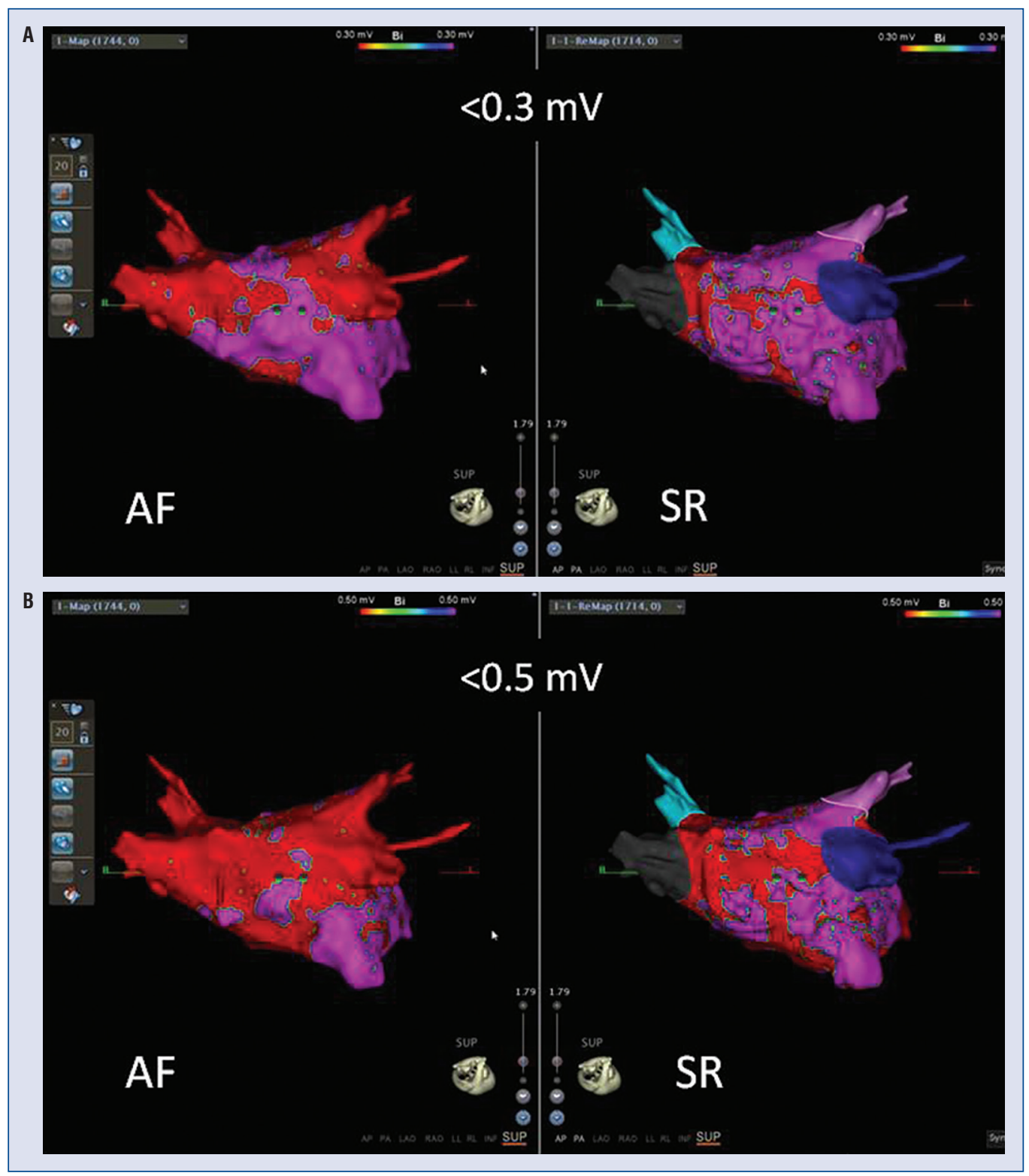

Figure 6. A. Comparison between atrial fibrillation (AF) and sinus rhythm (SR) maps for the $0.3 \mathrm{mV}$ threshold. Superior projection. Patient 20; B. Comparison between AF and SR maps for the $0.5 \mathrm{mV}$ threshold. Superior projection. Patient 20. In both images, an appropiate match in low voltage area (LVA) location is observed, although LVA extension is greater in the AF map.

lished to define AF substrate invasively. As an advantage, it does not require additional approaches or catheters and does not pose an additional risk to patients who, because of clinical criteria, undergo this therapeutic approach. Most authors consider these LVAs as surrogates of fibrosis $[15,16]$. In the present study, it was decided to use $0.3 \mathrm{mV}$ and $0.5 \mathrm{mV}$ as cutoffs for low voltage because there is a study [17] in which a cut-off point between $0.3 \mathrm{mV}$ and $0.6 \mathrm{mV}$ is proposed based on the histopathological study of acute and chronic ablation lesions performed in pig myocardium and because the most widely used voltage value in literature is $0.5 \mathrm{mV}$. Nevertheless, herein, there was no clear validation of a threshold that we have to consider fibrosis. Some authors have adopted a statistical approach, considering the $5^{\text {th }}$ percentile of all mapping points as a threshold for abnormal tissue. For example, Kapa et al. [18] proposed a threshold of $<0.2 \mathrm{mV}$ for atrial fibrosis in the posterior wall and in the area between the PV and LA and $<0.45 \mathrm{mV}$ for the rest of the atrium based on mapping 20 patients 


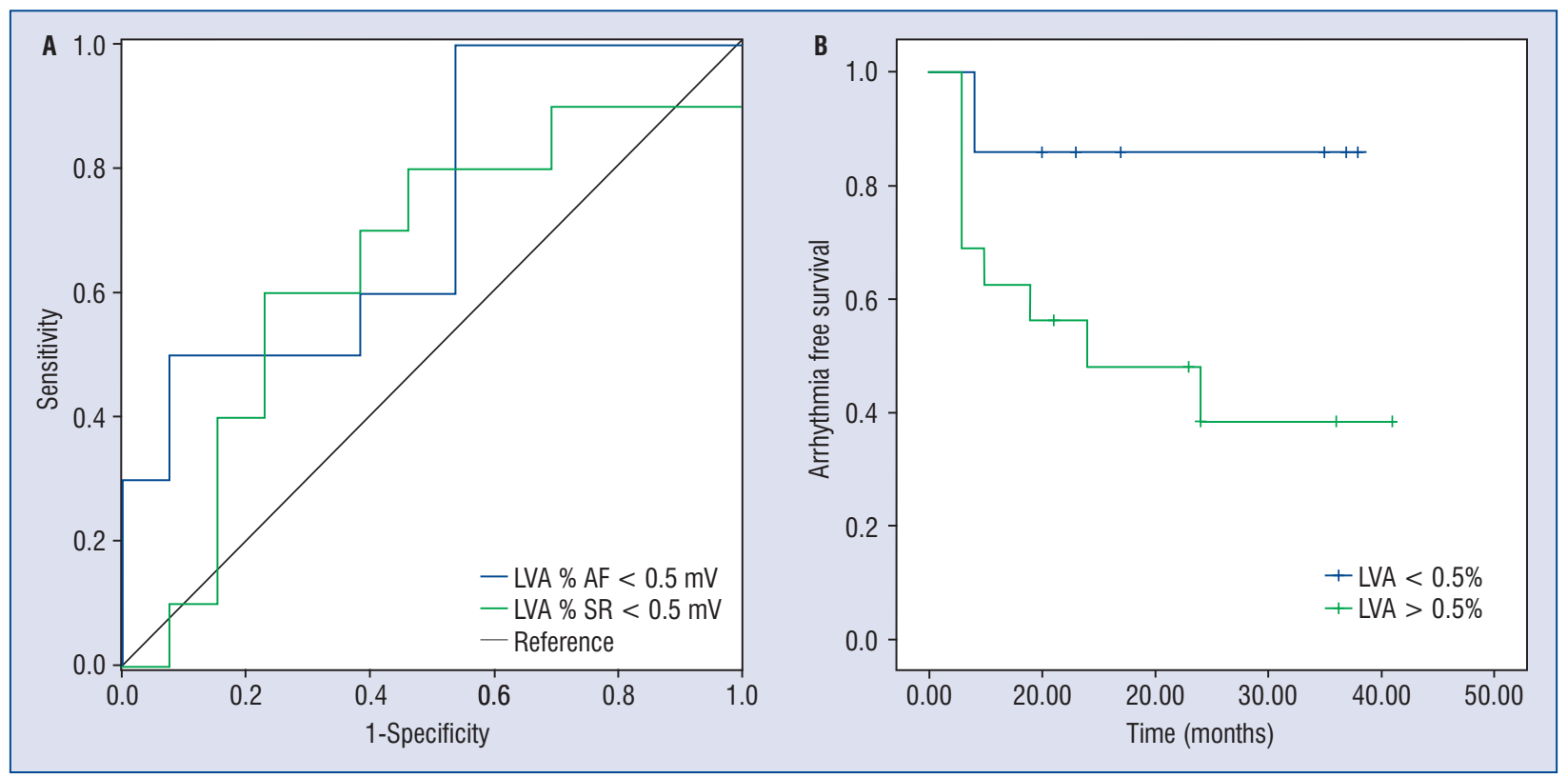

Figure 7. A. Receiver operating characteristic curves; B. Low voltage area (LVA) Kaplan-Meier arrhythmia free survival, excluding blanking period. LVA indicates low voltage areas $<0.5 \mathrm{mV}$ in atrial fibrillation maps.

with paroxysmal AF, 10 of whom had previous PVI. Another study [19] was carried out in patients with left accessory pathway ablation, some of whom had additional AF. In patients without AF, 95\% of the electrogram voltage was greater than $0.38 \mathrm{mV}$, so they defined a fibrosis voltage threshold of $0.4 \mathrm{mV}$. Other studies have been carried out in patients with supraventricular tachycardia undergoing LA mapping: Saghy et al. [20] established a cut-off point between fibrotic and healthy tissue of $0.5 \mathrm{mV}$ in a study of 9 patients, while Yagashita et al. [21] proposed $1.17 \mathrm{mV}$ in a study of 6 patients. In the literature, the most widely used voltage value is $0.5 \mathrm{mV}$, but it may not be a threshold sensitive enough to identify areas with arrhythmogenic potential. Several studies [7-12] have assessed the usefulness of individualized ablation guided by LVA, and most of them have used the $0.5 \mathrm{mV}$ threshold. In nearly all of them, LVA ablation has shown favourable results. However, these studies have important methodological differences, some of them in mapping that could influence LVA determination. These differences include mapping catheter electrode spacing, electrode size, tissue contact, signal filtering, map number of points and heart rhythm during mapping. In the present study, a multielectrode catheter with an electrode size of $1 \mathrm{~mm}^{2}$ was used, similar to that used in the studies of Yang et al. [22] and Jadidi et al. [10], while the rest of the studies used larger catheters. In healthy tissue, large catheters can cause a higher voltage record, whereas in areas with some fibrosis, a voltage summation of healthy and fibrotic voltages can result in lower amplitude signals [23]. Our mapping catheter, despite not having a direct contact force measurement, does have a tool that confirms proper contact before signal recording of a point. Adequate and consistent tissue contact is essential to avoid underestimation of endocardial signal voltage. Regarding the map number of points, the present work presents the advantage of high-density mapping. All maps presented more than 400 points, with a mean number of points in AF maps of 3428 and in SR maps of 2319 points, much higher than the average number of points from previous studies (100-200 points) with a maximum in Jadidi's study [10] with 1024 points.

The main strength of the present work lies in its objective: comparison of LVA according to map rhythm. In none of the exposed studies, was mapping carried out in both rhythms in the same patient. The present results show that for a certain voltage threshold, LVA extension is greater in AF maps than in SR maps. Another finding in the same vein was that the percentage of points below both thresholds was higher in AF maps than in SR maps. Yagishita et al. [24] obtained a similar result to the current one: the mean voltage in $\mathrm{AF}$ was lower than that in SR when mapping both rhythms in the same patient. Furthermore, they established that for the 
$0.5 \mathrm{mV}$ threshold, the number of LVA in AF was greater than that in SR, which supports the present results; however, those findings do not provide information on the extent of LVA. Since fibrosis is defined histologically and cannot be modified depending on heart rhythm, these data support that acquisition mapping rhythm implies a variation in the voltage recorded at each endocardial point. In fact, as early as 2003, the Ndrepepal et al. [25] group assessed the mean voltage in the left and right atrium in $\mathrm{SR}$ and $\mathrm{AF}$ and observed that the voltage was significantly reduced when mapping was in AF. In addition, they observed that areas with the shortest AF length cycle had a greater voltage difference between $\mathrm{AF}$ and $\mathrm{SR}$, suggesting a possible effect of rapid and disorganized depolarization on collected voltages. More recently, a Spanish study [26], carried out with a very accurate methodology, goes further and compares voltages of selected points in SR maps and in arrhythmia maps (in some cases AF and in others atrial flutter). The authors established that a value of $0.5 \mathrm{mV}$ in $\mathrm{SR}$ maps corresponds to $0.38 \mathrm{mV}$ in atrial flutter maps and to $0.31 \mathrm{mV}$ in AF maps. With these data and with those obtained in the current work, it was considered necessary to establish different voltage thresholds depending on the rhythm in which mapping is performed. Furthermore, these thresholds should be generalized to scientific studies that evaluate LVA ablation impact. Additionally, parameters and tools for mapping should be standardized so they can be properly compared and generalized to clinical practice in case of favourable results.

Since LVA was less extensive in SR maps, the SR LVA location to the AF LVA location was compared. The qualitative scale used did not allow comparison if there were no LVA areas in SR maps. It was observed that in most patients, LVA in SR was present in maps in AF. From our point of view, these data indicate that LVA in SR probably corresponds to fibrosis, but it does not allow us to determine if the $0.5 \mathrm{mV}$ threshold supposes an overestimation of fibrosis zones in $\mathrm{AF}$ or an underestimation of these areas in SR.

With respect to postablation clinical evolution, it must be emphasized that the present data are only descriptive and exploratory for further investigations. If LVA is considered as a marker of fibrosis and therefore as a possible factor influencing postablation outcomes, it was found, for the $0.5 \mathrm{mV}$ threshold, a significant relationship between recurrences and absolute LVA percentage $(\mathrm{p}=0.019)$. This relationship could not be detected in SR, of which results are not significant. Although not clearly stated, it is possible that if the patient sample had been more numerous, extension of LVA in $\mathrm{AF}$ would have been a factor clearly related to arrhythmic recurrences in postablation follow-up.

\section{Limitations of the study}

This study had few patients, although the sample number is similar to many previous publications that address atrial EAM. Areas in AF and SR were both measured manually, which may cause operator-dependent variations. However, intraclass correlation coefficients show adequate interoperator agreement. Because of this finding, this factor influence seems to play a minor role. The study was not designed and does not have a sufficient sample size to assess evolutionary data; thus, the results obtained are only exploratory.

\section{Conclusions}

Using the same voltage thresholds, LVA extension in AF is greater than in SR for patients with persistent AF. The location of LVA in SR is present in $\mathrm{AF}$ in most patients. These findings provide arguments for defining a different atrial fibrosis threshold based on EAM rhythm.

\section{Ethics and consent}

The present study was approved by the ethics committee of the Hospital La Fe, Valencia, Spain. All patients participating in the study signed informed consent.

\section{Availability of data and materials section}

The datasets used and analysed during the current study are available from the corresponding author on reasonable request.

\section{Conflict of interest: None declared}

\section{References}

1. Kottkamp H, Schreiber D. The substrate in "early persistent" atrial fibrillation: arrhythmia induced, risk factor induced, or from a specific fibrotic atrial cardiomyopathy? JACC Clin Electrophysiol. 2016; 2(2): 140-142, doi: 10.1016/j.jacep.2016.02.010, indexed in Pubmed: 29766862.

2. Coumel P. Cardiac arrhythmias and the autonomic nervous system. J Cardiovasc Electrophysiol. 1993; 4(3): 338-355, doi: 10.1111/j.1540-8167.1993.tb01235.x.

3. Mahnkopf C, Badger TJ, Burgon NS, et al. Evaluation of the left atrial substrate in patients with lone atrial fibrillation using delayed-enhanced MRI: implications for disease progression and response to catheter ablation. Heart Rhythm. 2010; 7(10): 1475-1481, doi: 10.1016/j.hrthm.2010.06.030, indexed in Pubmed: 20601148. 
4. Li D, Melnyk P, Feng J, et al. Effects of experimental heart failure on atrial cellular and ionic electrophysiology. Circulation. 2000; 101(22): 2631-2638, doi: 10.1161/01.cir.101.22.2631, indexed in Pubmed: 10840016.

5. Hsia HH, Callans DJ, Marchlinski FE. Characterization of endocardial electrophysiological substrate in patients with nonischemic cardiomyopathy and monomorphic ventricular tachycardia. Circulation. 2003; 108(6): 704-710, doi: 10.1161/01. CIR.0000083725.72693.EA, indexed in Pubmed: 12885746.

6. Hutchinson MD, Gerstenfeld EP, Desjardins B, et al. Endocardial unipolar voltage mapping to detect epicardial ventricular tachycardia substrate in patients with nonischemic left ventricular cardiomyopathy. Circ Arrhythm Electrophysiol. 2011; 4(1): 49-55, doi: 10.1161/CIRCEP.110.959957, indexed in Pubmed: 21131557.

7. Rolf S, Kircher S, Arya A, et al. Tailored atrial substrate modification based on low-voltage areas in catheter ablation of atrial fibrillation. Circ Arrhythm Electrophysiol. 2014; 7(5): 825-833, doi: 10.1161/CIRCEP.113.001251, indexed in Pubmed: 25151631.

8. Wang XH, Li Z, Mao JL, et al. A novel individualized substrate modification approach for the treatment of long-standing persistent atrial fibrillation: preliminary results. Int J Cardiol. 2014; 175(1): 162-168, doi: 10.1016/j.ijcard.2014.05.022, indexed in Pubmed: 24874911.

9. Cutler MJ, Johnson J, Abozguia K, et al. Impact of Voltage Mapping to Guide Whether to Perform Ablation of the Posterior Wall in Patients With Persistent Atrial Fibrillation. J Cardiovasc Electrophysiol. 2016; 27(1): 13-21, doi: 10.1111/jce.12830, indexed in Pubmed: 26515166.

10. Jadidi AS, Lehrmann H, Keyl C, et al. Ablation of persistent atrial fibrillation targeting low-voltage areas with selective activation characteristics. Circ Arrhythm Electrophysiol. 2016; 9(3), doi: 10.1161/CIRCEP.115.002962, indexed in Pubmed: 26966286.

11. Yamaguchi T, Tsuchiya T, Nakahara S, et al. Efficacy of left atrial voltage-based catheter ablation of persistent atrial fibrillation. J Cardiovasc Electrophysiol. 2016; 27(9): 1055-1063, doi: 10.1111/jce.13019, indexed in Pubmed: 27235000.

12. Blandino A, Bianchi F, Grossi S, et al. Left atrial substrate modification targeting low-voltage areas for catheter ablation of atrial fibrillation: a systematic review and meta-analysis. Pacing Clin Electrophysiol. 2017; 40(2): 199-212, doi: 10.1111/pace.13015, indexed in Pubmed: 28054377.

13. Qureshi NA, Kim SJ, Cantwell CD, et al. Voltage during atrial fibrillation is superior to voltage during sinus rhythm in localizing areas of delayed enhancement on magnetic resonance imaging: An assessment of the posterior left atrium in patients with persistent atrial fibrillation. Heart Rhythm. 2019; 16(9): 1357-1367, doi: 10.1016/j.hrthm.2019.05.032, indexed in Pubmed: 31170484 .

14. Yamaguchi T, Tsuchiya T, Fukui A, et al. Impact of the extent of low-voltage zone on outcomes after voltage-based catheter ablation for persistent atrial fibrillation. J Cardiol. 2018; 72(5): 427-433, doi: 10.1016/j.jjcc.2018.04.010, indexed in Pubmed: 29807864.

15. Kottkamp H. Human atrial fibrillation substrate: towards a specific fibrotic atrial cardiomyopathy. Eur Heart J. 2013; 34(35): 2731-2738, doi: 10.1093/eurheartj/eht194, indexed in Pubmed: 23761394 .
16. Callans DJ, Ren JF, Michele J, et al. Electroanatomic left ventricular mapping in the porcine model of healed anterior myocardial infarction. Correlation with intracardiac echocardiography and pathological analysis. Circulation. 1999; 100(16): 1744-1750, doi: 10.1161/01.cir.100.16.1744, indexed in Pubmed: 10525495.

17. Harrison JL, Jensen HK, Peel SA, et al. Cardiac magnetic resonance and electroanatomical mapping of acute and chronic atrial ablation injury: a histological validation study. Eur Heart J. 2014; 35(22): 1486-1495, doi: 10.1093/eurheartj/eht560, indexed in Pubmed: 24419806.

18. Kapa S, Desjardins B, Callans DJ, et al. Contact electroanatomic mapping derived voltage criteria for characterizing left atrial scar in patients undergoing ablation for atrial fibrillation. J Cardiovasc Electrophysiol. 2014; 25(10): 1044-1052, doi: 10.1111/jce.12452, indexed in Pubmed: 24832482.

19. Lin Y, Yang B, Garcia FC, et al. Comparison of left atrial electrophysiologic abnormalities during sinus rhythm in patients with different type of atrial fibrillation. J Interv Card Electrophysiol. 2014; 39(1): 57-67, doi: 10.1007/s10840-013-9838-y, indexed in Pubmed: 24113851.

20. Saghy L, Callans DJ, Garcia F, et al. Is there a relationship between complex fractionated atrial electrograms recorded during atrial fibrillation and sinus rhythm fractionation? Heart Rhythm. 2012; 9(2): 181-188, doi: 10.1016/j.hrthm.2011.09.062, indexed in Pubmed: 21946341.

21. Yagishita A, Sparano D, Cakulev I, et al. Identification and electrophysiological characterization of early left atrial structural remodeling as a predictor for atrial fibrillation recurrence after pulmonary vein isolation. J Cardiovasc Electrophysiol. 2017; 28(6): 642-650, doi: 10.1111/jce.13211, indexed in Pubmed: 28387462.

22. Yang G, Yang B, Wei Y, et al. Catheter ablation of nonparoxysmal atrial fibrillation using electrophysiologically guided substrate modification during sinus rhythm after pulmonary vein isolation. Circ Arrhythm Electrophysiol. 2016; 9(2): e003382, doi: 10.1161/ CIRCEP.115.003382, indexed in Pubmed: 26857907.

23. Anter E, Tschabrunn CM, Josephson ME. High-resolution mapping of scar-related atrial arrhythmias using smaller electrodes with closer interelectrode spacing. Circ Arrhythm Electrophysiol. 2015; 8(3): 537-545, doi: 10.1161/CIRCEP.114.002737, indexed in Pubmed: 25792508.

24. Yagishita A, DE Oliveira S, Cakulev I, et al. Correlation of left atrial voltage distribution between sinus rhythm and atrial fibrillation: identifying structural remodeling by 3-D electroanatomic mapping irrespective of the rhythm. J Cardiovasc Electrophysiol. 2016; 27(8): 905-912, doi: 10.1111/jce.13002, indexed in Pubmed: 27135965.

25. Ndrepepa G, Schneider MAE, Karch MR, et al. Impact of atrial fibrillation on the voltage of bipolar signals acquired from the left and right atria. Pacing Clin Electrophysiol. 2003; 26(4 Pt 1): 862-869, doi: 10.1046/j.1460-9592.2003.t01-1-00151.x, indexed in Pubmed: 12715847.

26. Rodríguez-Mañero $M$, Valderrábano $M$, Baluja $A$, et al. Validating left atrial low voltage areas during atrial fibrillation and atrial flutter using multielectrode automated electroanatomic mapping. JACC Clin Electrophysiol. 2018; 4(12): 1541-1552, doi: 10.1016/j.jacep.2018.08.015, indexed in Pubmed: 30573117. 